\title{
Innovation and Reflection: User Thinking of News Production Process in the Era of Smart Media
}

\author{
Hongyan Cong ${ }^{1 *}$, Yue Xin ${ }^{2}$ \\ ${ }^{1}$ School of Education, Northwest Normal University, ${ }^{1,2}$ School of New Media Art, Xi'an Polytechnic University , Xi'an \\ , Shaanxi, 710048, China \\ *Corresponding author. Email: 820760223@qq.com
}

\begin{abstract}
Under the global trend of active deployment of intelligent development, the journalism industry accurately grasps the opportunities of the times, uses intelligent high-tech to break resource barriers, reconstruct the news production business chain, innovate the human-machine collaboration news production process, and form a news production centered on user thinking idea. In this paper, the advantages and problems of the new intelligent news production mode are summarized from the aspects of news collection, production and distribution, by combing the application of various intelligent high-tech based on user thinking in the news production process. And then, further reflect on how to optimize the communication effectiveness in the era of smart media, make the best use of various smart technology materials, not only accurately convey news themes, but also meet the needs of users to the greatest extent, and achieve zero-distance and efficient "communication" between news and users.
\end{abstract}

Keywords: Intelligent media era, news production, user thinking, innovative reflection

\section{创新与反思：智媒时代新闻生产流程的用户思维 从红艳 ${ }^{1 *}$, 辛月 ${ }^{2}$}

${ }^{1}$ 西北师范大学教育学院, ${ }^{12}$ 西安工程大学新媒体艺术学院, 陕西西安 710048

*通讯作者. 由阝箱: 820760223@qq. com

摘要:

在全球积极布局智能化发展的浪潮下，新闻业精准把握时代机遇，借助智能高新技术打破资源壁垒，重构新闻 生产业务链，创新人机协作新闻生产流程，形成以用户思维为核心的新闻生产理念。本文分别从新闻的采集、 制作、分发层面，通过梳理各种智能化高新技术基于用户思维在新闻生产流程中的应用，归纳新兴智能化新闻 生产模式的优势及问题，进一步反思如何优化智媒时代的传播效力，使各种智能科技物尽其用，在精准传达新 闻主旨之余, 最大程度满足用户需求, 实现新闻与用户之间的零距离高效 “沟通”。

关键词: 智媒时代; 新闻生产; 用户思维; 创新反思

\section{1. 前言}

智媒时代的到来，促使新闻生产由受众思维向用 户思维转变, 传统主流媒体在新闻生产和信息传播方 面不断寻求最佳突围路径, 重构新闻信息在民众心目 中的地位。现如今, $5 \mathrm{G}$ 技术、人工智能 (AI)、大数 据、“云”端化以及虚拟现实等高新技术的应用，革
新新闻生产方式，一次次突破新闻边界，为用户带来 全新的新闻消费体验。但以此同时，智能科技在媒介 生态系统中的介入，也滋生出例如信息茧房、新闻伦 理、报道主旨偏颇、阶层固化等问题。基于此，新闻 传播各环节主体需积极配合，完善智能化技术在媒体 行业的有机融合与运作，做技术的主人，进而优化未 来新闻生产的发展方向。 


\section{2. 智媒时代新闻生产流程的用户思维}

智媒时代的一个重要特征就是简化用户的生活 方式，即时提供满足用户需求的服务，使其在短时间 内迅速掌握相关内容信息。这一特征与新闻业相结合, 驱使新闻工作者在对新闻的采集、制作以及分发等方 面必须具备用户思维，根据不同用户的特点、喜好， 制定个性化、细分化的新闻资讯，以满足用户的多方 位需求。

\section{1. 基于万物皆媒的多维度新闻采集}

新闻采集即是对新闻素材的发现、收集以及整理, 优质新闻的生成需要有优质的新闻线索材料作为支 撑, 为用户传递内容新颖、高价值的信息。在传统媒 体对新闻的采集过程中, 新闻编辑或者记者要查看海 量的资料并进行实地考察, 再将搜集到的资料归纳整 理, 不仅延长新闻生产周期, 还降低新闻生产效率。 且在记者对数据梳理和信息整合过程中, 常常会受到 主观意识的影响, 从而导致新闻的客观性出现偏差。 如今的智媒时代, 缓解传统媒体新闻采集的诸多问题, 万物皆媒的特性打破了新闻记者单向度采集新闻的 常规模式, 呈现多维度采集趋势, 而技术的发展, 恰 恰给予了其强有力的支撑。

\section{1. 1. 被动采集的区块链技术}

2008 年, 自称日裔美国人的中本聪（Satoshi Nakamoto）首次在其发表论文的《比特币：一种点对 点式的电子现金系统》中提出区块链这一概念 ${ }^{[1]}$ 。区 块链技术综合了计算机工程、密码学、数学、经济学 等多种学科, 以多个个体普遍认同的共识算法形成信 任机制, 采用个体用户 “分布式记账” 的方式搜集信 息, 具有去中心化、精确性、不可篡改性、匿名性等 特点, 最终构建一个安全稳定、同步共享的数据库。 新闻领域对该技术的应用, 可以形成一个透明公开的 “新闻公告栏”, 为新闻的采集以及新闻的真实性提 供了保障, 之所以称之为被动采集, 是因为它依赖于 个体用户对信息的主动上传, 才能搜集到相关的新闻 线索。新冠肺炎疫情期间, 健康码的推出为我国的复 产复工提供了坚实的基础。利用区块链技术, 通过用 户在平台输入自己的实名身份信息或者扫码的方式, 链上智能合约根据健康验证规则计算该用户的健康 状态, 最终生成健康码并在链上存证 ${ }^{[2]}$, 与用户行动 轨迹的多种数据源有效对接。各个地区可共享验证结 果, 并且可以交叉验证, 使得健康验证结果更加精确。 健康码的普及保障了复工复产的安全性, 为新闻疫情 报道提供了安全可靠的数据资源, 新闻真实性得到保 障。
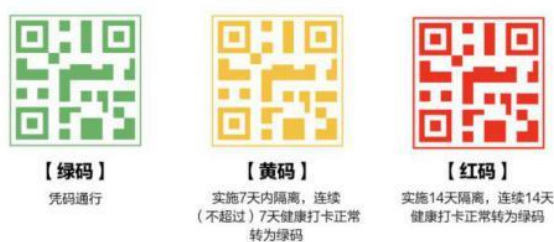

\section{防控疫情人人有责}

图 1 健康码（来源：网络）

\section{1.2 主动采集的传感器技术}

传感器技术主要是将感应、监测到的信息进行相 应的转换和输出。在新闻采集环节, 它将传感器作为 主要信息搜集的载体, 大数据处理技术辅以支撑, 为 新闻报道提供丰富素材、篮选优质元数据。无人机作 为传感器的一种类型，被普遍应用到新闻采集过程中。 面对一些重大的灾情现场, 记者无法抵达现场的空间 领域, 进行现场画面的拍摄, 这时就可借助无人机对 灾情现场进行画面信息的采集，使新闻报道更加具有 真实感以及说服力。例如, 在此次新冠肺炎疫情重灾 区武汉建设火神山和雷神山医院时, 央视频客户端第 一时间开通慢直播通道 ${ }^{[3]}$, 借助摄像机以及无人机等 设备, 为民众呈现两所医院的建造过程, 为广大网友 提供了线上 “监工” 条件, 让流言不攻自破。此外, 还有一种智能传感器, 它主要对人的生理感觉变化

(心率、血压、脑电波等) 进行精准记录, 通过可视 化数据的呈现方式, 帮助记者深入挖掘人对待一件事 或事物的真实感受, 有助于记者对新闻报道的分析和 把控。2018 年两会期间, 新华网推出生物传感智能 机器人 Star, 记录政府工作报告时观众的情绪起伏, 从而生成了国内首条生理传感新闻 ${ }^{[4]}$, 使得政务之于 观众变得更加有温度。

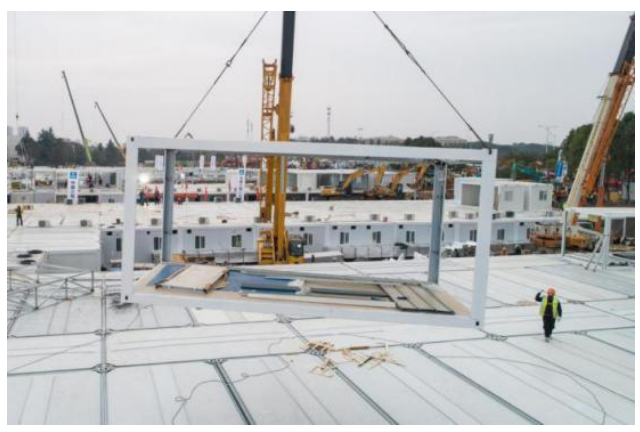

图 22 月 1 号拍摄的武汉火神山医院建设工地(来源: 新华社记者 程敏 摄)

\section{2. 新闻制作环节人与智能协同发展}

新闻制作是新闻生产流程中的核心环节, 如何将 海量的信息资源进行分类有序的排版与整理, 并且清 晰地向用户传达新闻主旨, 这是该环节需考虑的主要 问题。传统媒体时代，新闻制作只能依靠专业的新闻 
编辑完成, 工作量大, 出错率高, 生产周期长, 生产 数量少, 难以满足用户对多元化新闻的需求; 融合媒 体时代, 互联网的发展降低了新闻制作的准入门槛, 用户也可加入该环节, UGC (用户生成内容) 新闻丰 富了新闻种类，打破了信息单向度传播模式，用户对 新闻掌握了一定的主导权以及发言权; 智能媒体时代, 人工智能进入大众视野并参与新闻制作，根据研发者 输入的程序形成固定的 “行为” 以及 “思维” 模式, MGC（机器生产内容）新闻在新闻的制作过程中呈现 准确率高、生产效率高等优点 ${ }^{[5]}$, 以配合新闻编辑以 及记者完成一些无需深度思考的新闻报道。

\section{2. 1. 写稿机器人丰富新闻制作版本}

“机器新闻写作” 首先基于一个庞大的数据库, 技术人员通过编写程序、优化算法与数据逐步提升机 器人对于数据库的理解能力, 经过一段时间的学习, 机器人具备强大的数据分析能力以及写作能力, 将人 工研读数据和编写新闻时间缩短至几秒钟, 可以达到 “全天候、不间断” 的资讯推送。早在 2001 年, 谷 歌为实现个性化新闻的推荐, 就开始尝试使用机器选 编新闻, 开创了机器人参与新闻编写的先河 ${ }^{[6]}$, 此后 国外的多家权威媒体, 例如路透社、《华盛顿邮报》、 《纽约时报》等纷纷开始加入此行列, 逐渐拓展了机 器人在新闻制作中的功能, 由校对、核查到编写新闻, 为解放人力资源提供了技术支持。2015年 9 月 10 日, 我国诞生首篇 “机器人新闻”, 新闻生成仅耗时 1 分 钟 ${ }^{[7]}$ 。Dreamwriter 的问世, 在缩短新闻制作时长、 清楚表达新闻主旨的同时, 还增加了许多新闻报道的 版本, 用户可针对自身情况, 在忙碌时选择精简版新 闻, 触及到陌生领域可选择民生版新闻, 对专业领域 知识熟知可选择研判预测版新闻, 使新闻内容传播更 加人性化。

\section{2. 2. 智能视频剪辑提升新闻制作效率}

继写稿机器人之后, 人工智能视频剪辑让视频新 闻有了更多的可能性。为了用户制作短视频更加方便、 精美且速成, 如今的视频剪辑软件弱化了其专业性, 提供了各式各样精美的配图配文资源包、片头片尾以 及水印模板, 还可以自动识别视频素材声音生成字幕 等等, 这一系列的功能都是科技智能化的体现。而当 机器人与视频剪辑相结合, 智能化将再度升级。Hao 球国际是一家体育互联网公司, 致力于为球迷提供赛 事直播以及赛事管理解决方案等。该公司在 2018 年 研发的人工智能视频剪辑可基于运动赛场内所发生 的事件进行智能识别并分析计算, 快速对赛场的经典 瞬间以及热点场景进行智能剪辑, 生成短视频新闻, 供第三方媒体进行转载, 扩大赛事传播力度。该技术 的应用, 丰富了体育赛事的播报形式, 用户可以快速 了解赛场上的精彩瞬间, 让体育新闻进入生活。

如今, 智能机器人对新闻制作的参与, 主要在体 育新闻领域以及财经新闻领域较为多见 ${ }^{[8]}$, 因为这两
类新闻有相对的固定性和较强的预见性, 实现自动化 写作的可操作性更强。有智能机器人对于数据收集及 分析的助力, 一方面可以保证新闻数据的客观性, 避 免新闻编辑或记者主观意识的介入; 另一方面, 可根 据数据的走向趋势把握未来的新闻市场形式, 为新闻 报道提供预测性意见。

\section{3. 算法推荐实现新闻个性化分发}

传统媒体时期，由媒体主导新闻分发的内容，根 据其主观意识对新闻信息价值的审视和判断决定内 容选取以及排版，大众只能通过报纸、广播、电视等 平台载体听到或看到政府及媒体想让我们了解的新 闻, 因此, 新闻内容相对统一且固定, 大众对新闻的 兴趣选择被忽略; 而由于互联网的兴起，新闻传播速 度快、范围广, 大大小小的新闻都可以通过这张“网” 推送给受众, 且 UGC 的加入更加丰富了信息量, 受众 的新闻兴趣度被重视起来, 知情权以及言论自由权进 一步得到保障。面对海量的信息，受众在选择变多的 同时，也经受着信息冗杂、信息污染、内容同质化等 问题的困扰。在此背景下，基于用户思维的个性化算 法推荐技术应用而生，将其应用到新闻分发的环节， 根据用户的关注以及喜好对新闻采取过滤机制, 进行 精准分发。智能化的服务模式为用户在新闻的选择上 节省了时间，使得生活变得更加方便快捷。

\section{3. 1. 大数据与 “云” 技术: 细化用户画像, 延伸信息价值}

大数据技术是通过对基于互联网背景下用户对 各种应用的使用产生的巨量数据进行处理以及分析, 从而赋予数据洞察实事、参与决策、优化流程的能力, 为新闻工作者提供更多有价值的信息。“云” 技术是 一种为方便用户对信息进行整合、存储、计算的技术 解决方案, 无需依赖个人终端就可自动完成数据的更 新, 并且可以在多个服务器上共享信息, 为用户按需 获取资源提供便利。在新闻分发过程中，一些软件在 开始界面就设置了各类选项用于兴趣推荐, 目的是为 用户提供更加优质的人性化服务; 此外, 大数据技术 可根据用户对互联网的使用痕迹，收集汶览、点赞以 及搜索等记录, 接着解析其关注点生成用户画像, 最 终将与其兴趣爱好相匹配的新闻资讯进行精准推送, 为用户节约了信息節选时间; “云” 技术基于多人访 问同一信息的共享机制以及同一信息差异化使用的 开放机制 ${ }^{[9]}$ ，使得信息价值最大化，简化了新闻分发 流程, 从而达到一劳永逸的效果。在大数据与 “云” 技术的赋权下，《今日头条》的内容分发算法已经服 务了全球的亿万用户; 瑞士的新闻机构 Tamedia 旗下 的个性化推荐算法 “Flint” 已经带来了 2.5 倍的点 击率 ${ }^{[10]}$; 疫情期间的 “云” 服务将智能化、无接触等 名词送入大众视野…… 


\section{3. 2. 新闻聊天机器人：无时差深入交流，迅 速了解新闻主旨}

另一种算法推荐是以人机对话的形式模拟人际 交流，让新闻分发在聊天中进行。媒体可以在自己的 客户端或者借助自媒体平台基于人工智能技术构建 一个可以聊天的智能机器人虚拟形象, 通过与用户情 感交互式的聊天方式, 捕捉关键词以及话语信息, 分 析其情感偏向和兴趣爱好, 为用户推送相应的新闻内 容。有些聊天机器人, 不仅会使用文字互动, 还可以 向用户发送语音以及表情包图片, 进一步打破了人机 之间的疏离感 ${ }^{[11]}$ 。此类 “聊天沟通式” 的新闻分发模 式, 一方面为用户对新闻的接收增加了沉浸感和交互 感, 拉近了媒体与用户之间的距离; 另一方面, 有些 信息需要用户参与互动才可以获得完整的新闻内容, 有助于用户理解新闻内容, 规避一目十行、断章取义 式新闻阅读习惯，让新闻主旨做到精准传达。2020 年新冠肺炎疫情爆发之后, BBC 新闻在 Facebook 上 推出 BBC Corona Bot, 用户可在聊天框内输入自己 想了解的有关英国疫情的相关新闻, 对面的机器就会 推送一些最新的相关资讯, 迅速获取英国任何城市的 疫情发展程度以及复工复产情况等。

\section{3. 智媒时代用户思维生产新闻的创新与反思}

从传媒时代到融媒时代再到智媒时代, 这不仅仅 是字面的改变, 更是媒体行业一次次脱胎换骨的生态 重塑, 在此过程中, 用户的感受逐渐被新闻工作者重 视, 并成为新闻生产流程中需要考虑的前提以及判定 新闻质量的标准。用户的需求是受年龄、学历、社会 经历、生活环境等多重因素影响的变量, 具有个性化、 多样化、易变化等特点。智媒时代, 技术赋权让新闻 生产流程中的用户思维在不断的更新迭代中深化, 新 闻信息推送的 “私人订制” 服务为用户带来了绝佳的 体验, 但任何事物都有两面性, 在享受技术带来便利 的同时也应反思其带来的冲击, 媒体如何平衡用户需 求和新闻价值之间的关系, 用户如何走出 “信息舒适 圈”, 机器的计算可以确保万无一失吗?

\section{1. 创新：打造全新的媒介世界}

\section{1. 1. 打破时间与空间的界限}

如今，以 $5 \mathrm{G}$ 技术为依托的互联网实现了信息接 收速度的显著提升, 重塑了一些新的新闻传播形态, 为更多的智能化设备更新与升级提供了增益效能。在 各种新兴的智能化设备中，最引人注目的当属 VR、 AR、MR 技术。VR 技术是虚拟现实 (Virtual Reality) 技术的简称，它利用计算机仿真技术生成一个三维动 态的虚拟世界, 可以让用户有 “穿越” 时空的感觉。 AR 技术是增强现实（Augmented Reality）技术的简 称, 它可以让虚拟世界与现实场景形成互动, 让用户 体验更加真实。MR 技术是混合现实 (Mixed Reality) 技术的简称，它的最高境界是可以将模拟环境与现实
世界形成天衣无缝的融合，很难被区分，不仅可以进 行互动，用户还需实时反馈信息。 $5 \mathrm{G}$ 技术以其超高 速率、超低延时、超大容量的优越特性, 使得 VR、 AR、MR 技术的画面数据传输更加快速、流畅、清晰, 虚拟信息与真实世界的交叉重叠, 让用户完全拥有属 于自己的时间轴和空间系 ${ }^{[12]}$, 可基于个人终端自主选 择接收与阅读新闻的时间, 并且形成更加沉浸式的深 度体验。2019 年颁布的美国网络新闻奖中, 一则名 为《Poisoned Cities, Deadly Border》(中毒的城市, 死亡的边界) 的新闻作品荣获 “杰出创新视觉与数字 辅助报道奖” ${ }^{[13]}$, 该作品重点报道了美国与墨西哥边 界新河（New River）附近的空气污染、水质污染以 及环境监管等问题。用户可以依据个人阅读习惯选择 1 分钟、 5 分钟等时长不等的作品从任何地方、任何 故事开始阅读, 报道采用了交互式图片、长短片视频 以及 360 度 VR 体验等方式，讲述因污染患病导致死 亡的居民的故事, 这种自主选择的沉浸式报道让读者 更加直观地了解当地居民的感受, 引起对环境保护的 重视。

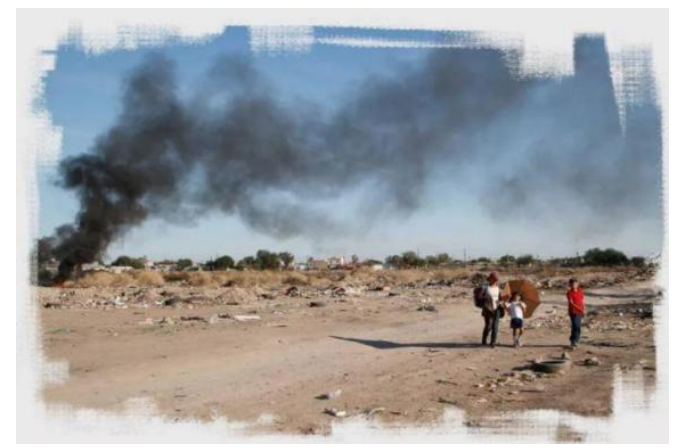

图 3 Poisoned Cities, Deadly Border (来源: 《沙 漠太阳报》官网)

\section{1.2. 重构用户记忆模式}

智媒时代的互联网为大众搭建了一个 “个人记忆 存储银行”, 每一个应用程序就是一个 “银行”, 我们 可以将自己喜爱或者有用的信息在相应的应用程序 中 “云存储” ${ }^{[14]}$, 这样的方式既为用户的个人终端设 备节省空间, 又可以安全有效地保障信息不会丢失。 有时人们会说: 互联网是有记忆的; 但有时又会提出: 互联网是没有记忆的。实则, 两句话针对的主语不同, 前者指的是用户个体的数据记忆, 后者指的是社会群 体的大脑记忆。任何人只要使用互联网, 都会留下相 应的数据痕迹, 以数据化的形式存在在网络空间里, 并且被永久保存。一方面, 数据记忆体现在用户可以 通过对软件的使用进行信息获取或者情绪宣泄, 来 “刷存在感”, 使其当下的状态被数据永久得保存, 之所以为 “永久”, 是因为即使日后删除, 数据也可 以对其进行恢复, 即便用户有时并不想这么做; 另一 方面, 面对不断更新的社会热点话题, 旧的热点事件 很难在社会群体的大脑记忆中留下挥之不去的深刻 印象, 因为大众必须将自己的热点库随时与他人保持 一致, 在新旧热点中来回切换, 才能拥有与他人社交 
的谈资。技术的发展的确赋予了用户更多的话语权, 但其永久保留的数据记忆则监督大众需要更加地谨 言慎行。

\section{2. 反思：智媒时代给用户带来的隐患}

\section{2. 1. 信息爆炸导致社交焦虑感}

各类社交软件的开发与上市，让用户与用户之间 形成一种 “云互动”，只要有一个共同爱好或者共同 话题, 大家就可以省去彼此相互了解的过程, 迅速成 为朋友。这样的交友方式看似轻易简便，实则却令大 众疲㤂不堪。智媒时代，信息都是 24 小时不间断的 全方位推送, 而根据社交需求, 我们必须紧跟时代潮 流，尽力去找与他人的谈资，作为自己的社交货币， 快速融入社交群体。因此面对如潮水般向我们涌来的 信息, 大众害怕错过, 疲㤂感与焦虑感也就随之而来。 此外, 还有一种线下的焦虑, 被称之为社交恐惧症。 用户可以在网络社交平台为自己构建专属人设, 该人 设相对完美, 具有开朗活泼, 善于交际等性格特点, 以博得更多网友的关注与喜爱。但在线下的社交关系 中, 离开了网络这层面具, 仿佛无形中缺失了一个体 面的开场白工具, 因此面对自己不熟悉的人, 往往会 表现得无所适从, 局促不安。换个角度来看, 焦虑感 是因为大家对自己都会有一定的要求, 不论是交友、 情感上的需求, 亦或是知识层面的需求, 如今信息爆 炸已成为不可阻挡的发展趋势, 合理、适当的焦虑会 让我们对新闻信息有更多的求知欲, 从而有利于自身 的发展。

\section{2. 2. 算法推荐造成信息茧房}

用户思维的提出与应用, 让新闻媒体更加关注千 人千面的个性化推荐方式, 而智媒时代的兴起, 则让 新闻内容的决定权交由算法, 让新闻价值的评判权交 由 “流量”。美国学者约瑟夫・克拉帕 (Klapper, J. T. ) 在研究受众对新闻的接受过程中提出选择性心理, 指 出大众只愿意接受自己感兴趣的、熟知的新闻内容, 并且会对与自己观念相斥、兴趣不符的新闻形成自我 防御机制, 自动选择不予接收。算法推荐技术虽然可 以为用户过滤冗余信息并投其所好, 但同时也使得用 户接受的信息同质化严重, 不能更新其观点和理论, 拒绝走出自我的舒适圈，掉入算法编织的“信息茧房” 之中。首先，长期对同类新闻的阅读会使用户的信息 面窄，视野固化，从而失去对事物的批判精神与思考 精神，形成 “单向度的人”; 其次，受众会和自己兴 趣、观点一致的人自动组成群体, 形成文化部落, 在 一次次强化其原有态度的过程中, 产生“回音室效应”, 易造成价值观偏执, 认知极端化; 此外, 长此以往地 屏蔽新信息、新事物, 使大众对于社会的认知产生很 大的两极分化状态, 加剧数字鸿沟。

\subsection{3. 利用隐私交换服务}

随着大数据技术的不断更新, 用户在不知不觉的 情况下, 信息就被自动识别和提取, 一举一动都被网 络 “监控”, 由此带来的隐私问题成为用户关注的焦 点。为实现新闻信息的 “私人订制” 服务, 在新闻的 采集、制作、分发流程中，几乎都会应用到大数据以 及云计算技术, 用户只要进入互联网, 其数据痕迹就 会被永久保留, 且由于当下智能化的不断升级, 一些 “黑科技” 甚至可以通过声音，识别用户正在交谈关 注的事物, 这些信息内容同样可以被录入数据库, 进 行后台分析以及共享。另一方面, 一些新闻客户端在 用户下载后会对用户设备的相册、媒体内容、通讯录、 移动网络等隐私信息进行强制读取, 获取权限, 逼迫 用户在 “被知情且同意” 的情况下签订 “霸王条约”, 若用户拒绝交出个人信息, 将无法正常使用该软件。 在这种被迫利用隐私换取服务的模式下，用户的地理 位置、社交关系、聊天记录等重要信息变得公开透明, 媒体通过在客户端 APP 的得到的数据进行频繁的交 换和共享，但用户却对个人隐私的用途及去向一无所 知。2018 年 3 月,美国最大的社交软件之一 Facebook, 被懪将其平台 8700 万用户的个人信息泄露给剑桥分 析公司 (Cambridge Analytica)，用以干预美国大选， 使得社交媒介平台失信于民, 造成了极其恶劣的影响 ${ }^{[15]}$ 。

\section{2. 4. 新闻内容价值的缺失}

智媒时代， $5 \mathrm{G}$ 技术、人工智能、虚拟现实等高 新技术被广泛应用于新闻生产的各个环节，使得新闻 不再 “枯燥严肃”, 沉浸感以及互动感的加入, 为用 户带来优质体验。但另一方面, 技术的发展也分散了 新闻内容的部分注意力, 重 “量” 而不重 “质” 的新 闻事件时有发生，当用户沉迷于技术带来的听觉、视 觉、触觉、嗅觉等享受时, 新闻内容的主要传达就会 被沉浸式的感官体验瓦解，从而错失新闻的关键信息， 造成新闻内容价值的缺失。此外, 写稿机器人在新闻 制作环节的参与, 虽然为新闻工作者提供了便利, 但 生产出的新闻价值还有待进一步提升。首先, 机器人 基于大数据分析是用户为新闻生产导向的, 但用户关 注的焦点有时可能不具有重要新闻价值, 从而发生新 闻种类失衡现象; 其次, 机器人会基于技术人员输入 的新闻模板进行新闻的编写, 涉及到体育、财经类等 侧重数据报道的新闻尚可进行简单的信息分析以及 排列组合, 但在面对一些需要进行深度报道、理性判 断的社会性话题新闻时, 机器人能力明显不足, 并不 能保证新闻是准确无误与万无一失, 因此滋生虚假新 闻。例如 2017 年 6 月, 美国《洛杉矶时报》发出一 条新闻消息: 加州 IslaVista 地区发生 6.8 级大地震。 随后官方又在 Twitter 上澄清, 该则消息为写稿机器 人 Quakebot 发出的自动推文, 这场地震发生时间实 际为 1925 年。 


\subsection{5. 机器难以计算人的复杂性}

人工智能在技术盛行的今天成为很多行业的宠 儿, 以其特有的高效率学习能力、连续性工作能力、 超快速计算能力以及智能化分析能力提高了各行各 业的生产力, 达到了事半功倍的工作效率。因此, 多 数行业劳动者为人工智能发展的迅猛势头所担忧, 人 工智能是否会全面取代人类工作, 占据生产力的核心 地位。事实上, 如今人工智能的发展正处于弱人工智 能阶段, 主要给予人类工作以辅助功能, 来改善我们 现有的、不足的技术条件。另一方面，人类具有人工 智能不具备的先天条件以及后天经历, 每个人在成长 过程中都会形成独有的世界观、人生观和价值观，且 人类的情感和思维是复杂多变的。目前的弱人工智能 阶段, 主要侧重于对用户使用网络的数据分析阶段, 而这样的分析之于人性来说, 实则是简单片面的, 大 数据监测到个人的兴趣爱好只是他千面中的一面, 且 以目前的技术条件来说, 它不可能涵盖人性的方方面 面。也许未来, 人工智能可能会发展到强人工智能阶 段甚至是超级人工智能阶段, 但如今就人类掌握的技 术来讲, 足以操控现在的人工智能。由此可见, 人工 智能目前只是可以解放一部分生产力, 但它仍然无法 全面取代人工生产力。

\section{4. 用户思维优化的多重路径}

在传统媒体阶段, 新闻内容的选择与发布由媒体 单方面决定, 受众处于被动接收新闻的地位; 而如今 的智媒时代, 媒体、用户、智能化机器共同加入新闻 制作流程, 用户思维的应用重构了新闻的生产以及传 播模式, 用户掌握了更多主动选择新闻的权利。根据 上文分析, 这样的新闻生产模式, 虽然带给我们诸多 便利, 但我们在享受之际也出现很多值得反思的问题。 用户对社交的需求将自己置入焦虑状态，而其选择性 心理又是造成信息茧房的根本因素; 媒体没有做好新 闻把关人的基本职责, 一味地迎合用户造成新闻价值 逐渐失衡; 技术条件限制造成用户隐私得不到保 障……显然出现问题的原因是多方共同作用的结果, 这也就意味着, 新闻生产流程用户思维的优化, 需要 多重路径协同运作。

\section{1. 媒体：人工干预技术}

虽然智能化技术的兴起大大减少了新闻工作人 员机械化、重复化的工作环节, 并且能够准确感知和 探测那些人力所不能及的空间领域, 为新闻生产助力, 但对于新闻内容的把关、社会與论的整体走向、社会 价值观的构建等, 媒体则肩负着不可替代的重要责任。

当今社会正处于弱人工智能阶段, 写稿机器人还 不具备对深度社会话题的报道能力, 且算法推荐易造 成信息茧房, 但新闻的价值在于报道真实客观的事件 以引起受众的普遍共鸣并引导受众构建正确的新闻 价值观, 所以媒体不能完全信任机器新闻写作以及算
法推荐技术，应在智能技术负责新闻 “准度” 的基础 上，对新闻的 “温度” 进行加工，引起用户对新闻内 容的关注度与共鸣情绪, 确保用户需求与新闻价值的 平衡。另一方面, 万物皆媒已经成为不可逆转的趋势, 但媒体不能被 “万物” 牵着鼻子走，应该建立 “媒融 万物” 的新观念, 掌握新闻传播的主导权, 让 “万物” 为媒体服务。在此过程中, 媒体首先要提高议程设置 能力, 在遵循新闻真实性和客观性的前提下, 主动抛 出社会问题及观点, 并巧妙设置新闻发布的先后顺序, 引导用户参与讨论, 随之扩大媒体影响力; 其次, 在 发布一些公共性或者政治性议题时, 要注重新闻内容 和分发渠道与个体用户之间的关联性, 以用户可以接 受的表达方式以及经常关注的新闻平台为主, 传达重 要思想。

媒体对智能技术生产新闻的干预和把关，可以促 进优质新闻的产生，为用户解决信息茧房的困扰，吸 收更加新鲜、多元的知识和观点。

\section{2. 用户: 提高个人媒介素养}

媒介素养是指大众在面对海量信息时所具有的 判断力、选择力、理解力、思辨力以及延伸力和创造 力 ${ }^{[16]}$ 。在如今的媒介环境中, 用户既充当着新闻内容 生产者的角色, 又充当着新闻信息享用者的角色, 提 高媒介素养, 则是对用户的新闻批判能力提出了更高 的要求。从新闻内容生产层面来说, 微博、微信、抖 音等平台的出现, 产生了一大批自媒体人, 他们生产 的内容主要是对自己生活的记录、对重大事件进行讨 论并发表观点以及对某专业领域进行知识技能的分 享等等, 在此过程中, 自我选题需积极向上且知识技 能含量丰富, 言论发表要符合中国特色社会主义核心 价值观, 培养良好的媒介素养习惯; 从新闻信息享用 层面来说，用户对信息的选择至关重要，信息茧房的 形成, 追其根本，是因用户惰性造成的。大众只愿意 选择自己熟知领域的信息, 对新的知识领域很少涉猎, 因为这个过程需要一定的时间。但这样的发展趋势, 容易造成社会两极分化严重, 不利于社会和个人的发 展, 因此, 要想打破封闭的自拟环境, 就必须克服自 己的惰性来拓宽视野, 提升自己对异质观点的包容性, 接纳不一样的声音。

\section{3. 技术：优化算法及平台}

智媒时代, 新闻信息的分发以个性化算法为主要 依据, 在各大媒体、社交平台针对每位用户进行精准 推送。除算法推荐有信息过滤机制外, 平台也对用户 信息接收起一定作用的影响。首先，我们不得不承认 算法推荐确实简化了我们寻找信息的过程, 为用户节 省了时间, 在此基础上, 优化算法的本质是需要培养 用户对于异质信息的接收能力。算法推荐在迎合用户 兴趣爱好之余, 还可以对此进行预测, 推送一些以低 时间成本就可以了解到新领域的内容, 主动拓宽用户 的知识面与喜好, 帮助用户了解外部更广阔的世界; 
此外, 在用户普遍认同的一些事物或观点内推送不同 的意见、声音, 让其意识到世界的多面性 ${ }^{[17]}$ 。其次, 对于媒体、社交平台的优化, 可以从界面引导以及社 会关系方面入手，利用各种奖励机制刺激用户，形成 强制的信息引导，来促进用户对信息的了解；此外， 社交平台的一大优势在于, 可通过大数据技术分析用 户的社会身份以及社交圈层, 进而将其社会关系中其 他用户的兴趣爱好作为资源进行推荐，同样有助于用 户发现自己新的兴趣点, 达到信息协同过滤的效果。

\section{5. 结语}

如今, 各大媒体新闻生产的智能化应用尚且处于 初步探索阶段, 在享受智慧媒体带来便捷的同时, 也 要逐步完善智能新闻生产机制, 促进媒体、机器、用 户三方的协同、融合式发展。未来, 在新闻行业生产 现有技术的基础上，进一步提升新闻的互动性、陪伴 性、精准性, 满足用户的新闻参与感, 从而为新闻行 业提供更加广阔的发展前景。

\section{项目基金:}

2021 年度西安市社科规划基金项目：全媒介视 阈下西安红色文化遗产视觉传播力提升研究 (WL184)

\section{REFERENCES}

[1] Satoshi N. Bitcoin: A peer-to-peer electronic cash system[EB/OL]. http://www.bitcoin.org/bitcoin.pdf.

[2] Asia-Pacific Blockchain Incubation Think Tank. "What are the applications and values of blockchain in fighting the epidemic?" [EB/OL]. https://www.sohu.com/a/413410564_120760722

[3] Southern Metropolis Daily. Cloud Supervisor and Slow Live behind the Fire Thunder Mountain: An emotional resonance between builders and netizens [EB/OL].https://www.toutiao.com/i678798265847 6442119/

[4] Lu Changchun, Liu Yingying. Report on the Integration of Artificial Intelligence into the Two Conferences: Product Innovation, Technology Application and Function Analysis [J]. New Media Research,2019,5(01):1-3+28.

[5] Wei Xiao, Zhang Lin. The development strategy of China's TV media in the $5 \mathrm{G}$ era $[\mathrm{J}]$. Young reporters,2020(03):68-69.

[6] Zhang Chen. Redefining the Age of Intellectual Media: Changing Trends and Innovative Elements of Content $2.0 \quad[\mathrm{~J}]$. Publishing Wide Angle.2019(09):36-39.
[7] Tencent Finance. August CPI rose 2.0\% year-on-year, hitting a 12-month high[EB/OL]. https://finance.qq.com/a/20150910/019573.htm

[8] Yuan Xin. Changes and reflections on news content production from the perspective of artificial intelligence $[\mathrm{J}]$. Wide-angle publishing, 2020(05):68-70.

[9] $\mathrm{Li}$ Weidong. the development trend of cloud communication and the opportunity of the times $[\mathrm{J}]$. news and writing,2020(06):5-13.

[10] Shi Anbin, Wang Peinan. Brand remolding of traditional mainstream media in intellectual media era [J]. Television research.2018(07):7-9.

[11] Wang Xiaopei, Ling Qian. "Talking about the news": redefining the way of news distribution by news dialogue robot $[\mathrm{J}]$. Modern communication (Journal of Communication University of China), 2017,39(12):166-168.

[12] Peng LAN. Differentiation and Overlapping: Time in the Mobile Age [J]. Journal of Northwest Normal University (Social Science Edition), 2020,57(05):21-28.

[13] Tencent.com All Media Group | 2019 U.S. Cyber News Awards Works Full Analysis[EB/OL]. https://new.qq.com/omn/20191026/20191026A04I HX00.html

[14] Peng LAN. future media ecology: disappearing boundary and reconstructed territory $[\mathrm{J}]$. modern communication (journal of communication university of China),2017,39(01):8-14+29.

[15] Fang Yan, Liu Ting. Ethical evaluation and reflection on algorithmic news in the era of artificial intelligence $[\mathrm{J}]$. Young journalists, 2019(21):30-31.

[16] Gao Yuan. Reflection and solution to the phenomenon of hot news quenching $[\mathrm{J}]$. Young reporters,2016(08):4-5.

[17] Peng LAN. Multiple factors leading to information cocoon house and the path of "breaking cocoon" [J]. Press,2020(01):30-38+73 